\title{
The Research on Transient Burning Rate of Solid Propellant by Digital Image Processing
}

\author{
Xin Peng ${ }^{1, a}$, Bing Liu ${ }^{2}$ \\ ${ }^{1}$ Photoelectronics Academy of National University of Defense Technology, 410005ChangSha, China \\ ${ }^{2}$ Aerospace Academy of National University of Defense Technology, 410005ChangSha, China
}

\begin{abstract}
In order to obtain the burn rate of the solid propellant that is the important parameter of transient burning, the new method named digital image processing is presented. In the article, the principle of digital image processing is analysed; The burning face of the sample in the each time is located according the image and the coordinates of the burning face is obtained. In experiment the transient burn rate is measured by digital image processing and the accuracy is acceptable.
\end{abstract}

\section{Introduction}

The transient burn rate is the important parameter in the research of the burning character, so the measure of the transient burn is very important ${ }^{[1]}$. At present, the target line method and the acoustic emission method is used to measure burn rate in the static state. The two method can not obtain the burn rate at each time and variation of burn rate in the process of the burning.

The digital image processing method can gather the image of the whole burning process and obtain the transient burn rate at frame per second. Moreover, the method can mend the impact of burn rate due to the unusual burning and can not disturb the experiment because of the non-contact.

\section{The principle and flow path of the digital image processing}

\subsection{The method principle of the digital image processing}

According to the technique of digital image process gather the image in the process of the burning and read the curve of burning face. The gray level of each pixel in the image can reflect the light intensity of corresponding position in the solid propellant. The image of the burning face contain the brighter dot, so can judge the critical curve and define the curve as transient burning position. Then, convert the critical curve coordinate to the physical coordinate and calculate the transient burn rate. The relationship between the two coordinates is as follow ${ }^{[2]}$ :

$$
\left[\begin{array}{l}
x \\
y
\end{array}\right]=k\left[\begin{array}{l}
u \\
v
\end{array}\right]
$$

$(x, y)$ is the physical coordinate, $(u, v)$ is the critical coordinate, $\mathrm{k}$ is the similarity coefficient.

\subsection{The flow path of the digital image processing \\ ${ }^{a}$ Corresponding author:Xin Peng: 540057112@qq.com}

\subsubsection{Gray}

The image of the burning process is RGB picture. Firstly, we change it to gray image. Because the most flame in the experiment is red, so we increase the weight of red gallery, the conversion relation is:

$$
f=0.8 \times R+0.1 \times G+0.1 \times B
$$

$\mathrm{R}, \mathrm{G}, \mathrm{B}$ is the three quantities of red .

\subsubsection{Adjust the uniformity of light}

Because the burning chamber is closed and the light on the solid propellant is uniformity, it is necessary to adjust the uniformity of light.

\subsubsection{Gauss high pass filter}

The Gauss high pass filter is used to get signal of the high frequency light and the flame, in order to locate the position of the burning face accurately.

\subsubsection{Bilinear expansion}

The burning face of the solid propellant is concave. The most slope of curve is $45^{\circ}$ and $135^{\circ}{ }^{[3]}$. In order to link the light mass of the burning face smoothly, the bilinear expansion is used.

\subsubsection{Binary}

In order to get the burning profile easy, it is necessary to translate gray image into binary image.

\subsubsection{Connection profile}

The profile of the binary image is the discrete light. The discrete light profile is connected line by line. 


\section{The experiment of transient burn rate measure}

\subsection{The device of the experiment}

The device of the experiment of transient burn rate measure include burning chamber(Figure 1), CCD camera, light, fire device(Figure 2). The propellant fire from the head face. The process of the burning is recorded by the camera. The transient burning rate is measured according to the distance of two frames of the burning face. At the same way, A group transient burning rate is measured. In other words, transient burning rates are measured at time interval of frame of per second.

The experimental sample(Figure 3) is a rich fuel solid propellant strip $(10 \mathrm{~cm} * 10 \mathrm{~cm} * 60 \mathrm{~cm})$. The sample is fixed on the fire device, then the sample is put in the burning room. The parallel light is set at the behind cameral in order to heightened the definition of the sample. The sample must parallel the ground in the process of burning. The face of sample must perpendicular to the optical axis in the process experiment.

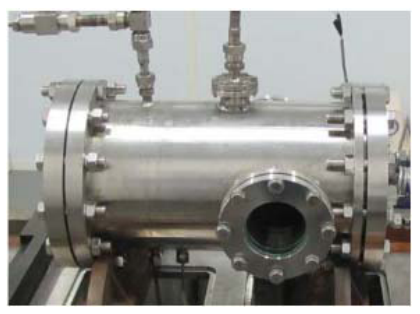

Figure 1. Burning chamber.

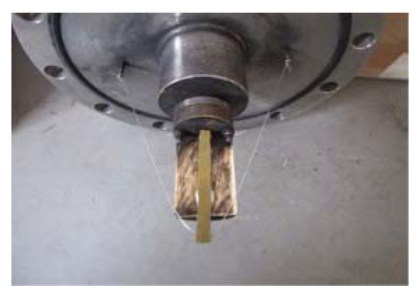

Figure 2. Fire device.

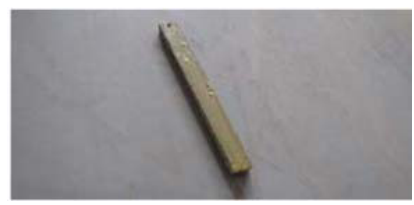

Figure 3. Sample.

\subsection{The result of the digital imagine processing}

The figure(4) to the figure(9) is the digital imagine processing result of $t 1$, the figure(10) to figure(11) is the result of $t 2$. The figure(8) to figure(9) is the effect picture of the location curve and the burning face curve. We can the green curve which is the location curve is good agreement with the burning face curve. the pixel coordinates of the burning face curve approximated to the pixel coordinates of the green curve. We can get the horizontal coordinate of the green curve and average it.
Then change it to physical coordinate that is the distance of burning face to the bottom face. We can the distance of two green curve between the adjacent two frame image. The time is the intervals per frame. The transient burning rate equal to the distance divided by the time.
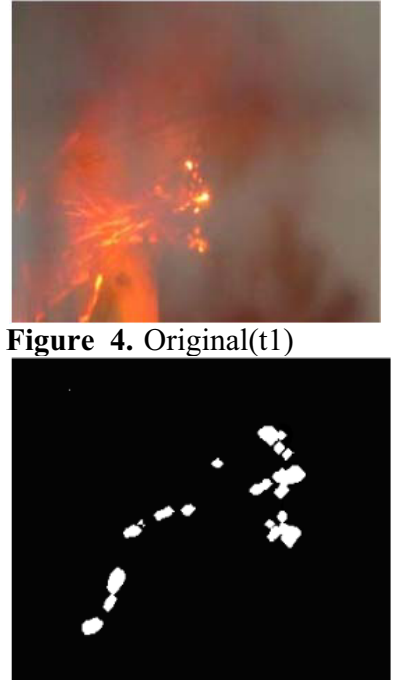

Figure 6. bilinear expansion(t1)

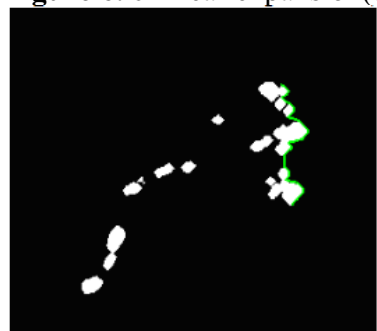

Figure 8. connection profile (t1). Figure 9. Consistent state(t1).

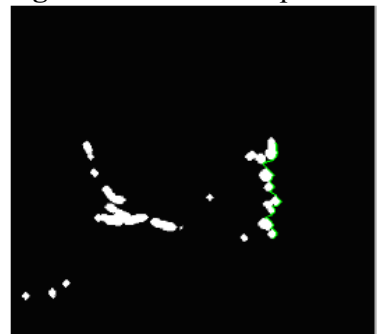

Figure 10. connection profile (t2) .
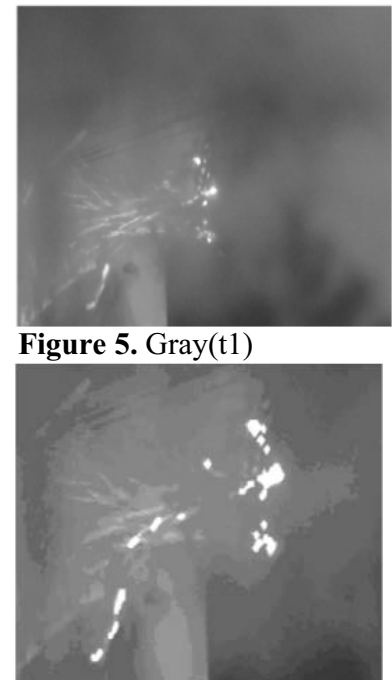

Figure 7. binary image $(\mathrm{t} 1)$.
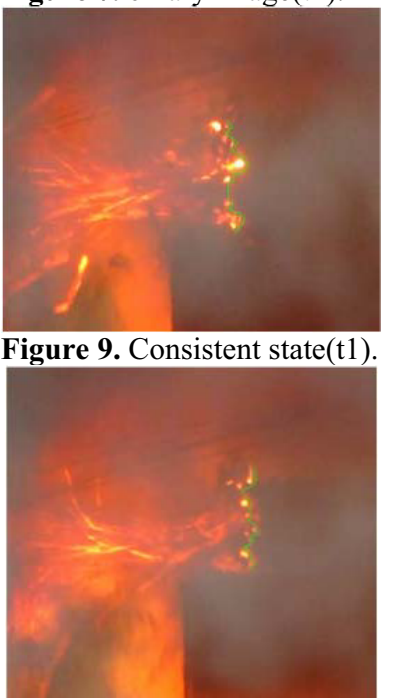

Figure 11. Consistent state (t2).
The averaged rate is equal that the length of the sample strip divide the time of total burning process. Compared the transient burning rate by the digital image processing to the averaged rate, there is small deviation. It is acceptable that the digital image processing is accurate method. The experimental parameters is show in the table(1) and the experimental result is show in table(2).

Table 1. Experimental parameters.

\begin{tabular}{|c|c|}
\hline Element & Parameter \\
\hline Pressure & $2 \mathrm{Mpa}$ \\
\hline Frame per second & 30 \\
\hline
\end{tabular}




\begin{tabular}{|c|c|}
\hline Similarity coefficient & 22.857 \\
\hline Average burning rate & $20.6 \mathrm{~mm} / \mathrm{s}$ \\
\hline
\end{tabular}

Table 2.Experimental result.

\begin{tabular}{|c|c|c|c|}
\hline & $\begin{array}{c}\text { Pixel } \\
\text { location }\end{array}$ & $\begin{array}{c}\text { Transient } \\
\text { burning rate }\end{array}$ & Deviation \\
\hline $\mathrm{T} 1$ & 494.3786 & $\begin{array}{c}21.8964 \\
\mathrm{~mm} / \mathrm{s}\end{array}$ & $6.3 \%$ \\
\hline $\mathrm{T} 2$ & 511.4157 & $\begin{array}{c}22.1198 \\
\mathrm{~mm} / \mathrm{s}\end{array}$ & $7.4 \%$ \\
\hline $\mathrm{T} 3$ & 528.0257 & $\begin{array}{c}21.5753 \\
\mathrm{~mm} / \mathrm{s}\end{array}$ & $4.7 \%$ \\
\hline $\mathrm{T} 4$ & 545.2781 & $\begin{array}{c}22.4050 \\
\mathrm{~mm} / \mathrm{s}\end{array}$ & $8.7 \%$ \\
\hline
\end{tabular}

\section{Conclusion}

We can get the transient burning rate by the digital image processing. The method is verified by experiment. Although the transient burning rate is not continuous, it can reflect the the burning character well.

\section{References}

1. Wei Zha, Hui Zhu, Ding You Fang, A Study on Mesurement of Fuel rich Propellant Combustion Property at Low Pressure, Energetic Materials, 3,118 (1999)

2. Qi Feng Yu, Yang Shang. Videometrics Principles and Researcher, (2008)

3. Yu Q, Ding X, Lei Z, Optical measurement system for bridge displacement andvibration, The $3 \mathrm{rd}$ Int.Conf. On Experimental Mechanics SPIE, (2001) 